\title{
IHM de Suppléance Sensorielle Visuo-Tactile pour Aveugles et d'Intégration Sensorielle pour Autistes
}

\author{
HERVE SEGOND \\ Université de Strasbourg \\ STEPHANE MARIS \\ Fondation Sonnenhof \\ YVES DESNOS \\ Université de Strasbourg \\ PERRINE BELUSSO \\ Université de Strasbourg
}

\begin{abstract}
Résumé : Nos travaux portent sur les mécanismes perceptifs et cognitifs impliqués lors de procédures d'apprentissage destinées à l'utilisation de technologies innovantes dans le domaine de la suppléance sensorielle visuo-tactile appliquée à la prévention des troubles du développement chez l'enfant aveugle et l'aide à l'autonomie des personnes aveugles. Nos objectifs reposent sur la conception et l'implémentation d'un dispositif embarqué esthétiquement acceptable de suppléance sensorielle, permettant une utilisation routinière dans le cadre des activités de la vie quotidienne, grâce à la conception de liaisons non filaires entre les composants de ces dispositifs, la réalisation d'un dispositif aux éléments modulables en fonction de la population ciblée (bébés aveugles, enfants et adultes aveugles), et l'interfaçage avec un poste informatique de numérisation d'environnement urbain 3D. Cette approche constitue une étape ultime et essentielle permettant de faire enfin bénéficier, dans leur vie quotidienne, les personnes handicapées des avancées de la recherche dans ce domaine. De nouvelles perspectives innovantes d'utilisation de ces dispositifs sont proposées dans le domaine de la prise en charge thérapeutique en faveur des personnes autistes.
\end{abstract}

Mots clés : Suppléance Sensorielle, Réalité Augmentée, Cécité Visuelle, Autisme, Technologie pour la Santé et l'Autonomie.

Abstract: Our research focuses on perceptual and cognitive mechanisms involved in learning procedures in order to develop and validate innovating technologies in the field of health for blind people through the implementation of an embarked version of a Tactile Vision Sensory Substitution device (TVSS) with wireless connections between the three TVSS components (micro camera, coupling system and matrix of tactile stimulators), aesthetically acceptable and allowing an ecological use. Such a device could allow prevention of developmental disorders in blind infants, autonomy for blind people in everyday life (thanks to the implementation of a Human Machine Interface allowing connections between the embarked TVSS device and a Personal Computer with a software development presenting a 3D numerization of the subject's urban environment). A new perspective is proposed in favor of innovating taking care programs for autistic people. The main goal of this project is to allow disabled people to benefit from the scientifically demonstrated efficacy of TVSS devices.

Key words: Sensory Substitution, Augmented Reality, Blindness, Autism, Technology for Heath and Autonomy.

Les articles de JIPS sont publiés sous licence Creative Commons Paternité 2.0 Générique 


\section{INTRODUCTION}

L'objectif majeur de nos recherches est le développement et la validation de solutions technologiques pour répondre cliniquement et socialement aux problèmes majeurs de santé publique que constituent $a$. la prévention des troubles du développement chez l'enfant aveugle, $b$. l'aide à l'autonomie des personnes aveugles, et $c$. la prise en charge thérapeutique dans le domaine de l'autisme avec ou sans handicap visuel associé. Cet objectif s'inscrit dans une volonté de contribuer à l'amélioration de la qualité de vie des personnes atteintes de déficience sensorielle, motrice, intellectuelle et de troubles psychiques. La suppléance sensorielle constitue une voie heuristique $a$. d'investigation de la plasticité cérébrale et de l'apprentissage perceptif et cognitif, d'une part, et $b$. du développement des aides visuelles pouvant être apportées, d'autre part, permettant l'accès à des informations spécifiquement optiques aux personnes aveugles. En outre les perspectives de prévention et de prise en charge thérapeutique en faveur des personnes aveugles et/ou autistes constituent indéniablement un enjeu majeur en matière d'impact social et économique.

\section{SUPPLEANCE SENSORIELLE : ETAT DE L'ART}

Le concept de «substitution sensorielle» (Sensory Substitution) est relativement récent en neurosciences. Il se rapporte à la possibilité d'accéder à des informations optiques par l'intermédiaire d'une modalité sensorielle autre que la vision, en permettant une perception de type visuel. La substitution sensorielle désigne ainsi la capacité du système nerveux central de se saisir de "prothèses sensorielles » pour constituer, par l'apprentissage, une perception de type visuel. L'emploi du terme initial de « substitution sensorielle » est encore aujourd'hui largement répandu dans la littérature. Toutefois, nous lui préfèrerons celui de « suppléance sensorielle », proposé dans les années 1980 en France [Sampaio and Dufier 1988], permettant d'apporter une nuance qui n'existe pas dans la terminologie anglosaxonne. En effet, le terme de «substitution» sensorielle pourrait sous-entendre, au sens littéral (et de manière optimiste), un simple changement d'entrée sensorielle; l'entrée visuelle classique étant remplacée par une entrée hybride (e.g. caméra et stimulateurs sensoriels). Face aux différences existantes entre un dispositif de couplage sensori-moteur artificiel (i.e. prothèse sensorielle) et le couplage sensori-moteur visuel (i.e. au niveau de la résolution temporelle et spatiale), une simple «substitution » n'est pas réalisable. C'est en cela que l'usage du terme "suppléance » semble plus adéquat que celui de « substitution» au sens où il laisse entendre que les dispositifs ne comblent jamais pleinement un déficit, mais qu'ils permettent l'ouverture d'un nouvel espace de couplage sensori-moteur de l'homme avec le monde [Lenay, Gapenne, Hanneton, Marque and Genouel 2000]. L'étude de la suppléance sensorielle sur un versant comportemental a débuté dans les années 1960. Le principal objectif des recherches menées a été de fournir une information de type visuel, via un autre canal sensoriel, pour apporter une aide d'un nouvel ordre aux personnes aveugles concernant les possibilités de perception de l'environnement distant. La vision est décrite comme étant la modalité dominante dans la perception de l'environnement spatial, et l'on comprendra aisément que sa privation diminue l'information perceptible disponible sur le monde qui nous entoure. Cette privation va conduire à l'usage accru des autres modalités perceptives disponibles. L'audition est sollicitée, permettant une perception à distance, mais 
limitée aux objets sonores. Le toucher est d'autre part fortement sollicité, mais il s'agit d'une perception de contact et la proportion de l'espace pouvant être appréhendé à un instant donné est limitée compte tenu de l'étendue du champ perceptif, occasionnant ainsi une moins bonne performance que la vision dans le domaine spatial.

Le toucher reste néanmoins la modalité sensorielle susceptible de mieux suppléer la vision. Il existe, en effet, certains avantages à l'utilisation d'une entrée tactile plutôt que sonore dans une perspective de suppléance sensorielle : la stimulation de régions corporelles rarement sollicitées peut limiter l'interférence entre informations à traiter au sein d'une modalité sensorielle, réduisant ainsi la charge attentionnelle portée aux informations émanant de l'environnement (comme c'est le cas pour la modalité auditive qui est essentielle chez les personnes aveugles dans leur rapport au monde extérieur). Pour le toucher, la conversion d'une image en événement tactile ne nécessite pas de recodage (contrairement à une conversion en événement sonore - cf. dispositif de suppléance visuo-auditive) ; l'image peut être directement reproduite sur la surface de la peau. Enfin, le toucher permet, de même que la vision, l'accès à certaines propriétés des objets tels que la forme, la taille, la localisation, l'orientation... Les dispositifs électroniques de suppléance sensorielle visuoauditifs ou visuo-tactiles ont été conçus, avec le développement de l'électronique, en vue de pourvoir les aveugles en flux de stimulations susceptibles de pallier la forte réduction des flux sensoriels responsable chez les aveugles de leurs difficultés perceptives et motrices. Les systèmes visuo-auditifs de suppléance sensorielle (guides ultrasoniques tels que le Sonicguide et le Trisensor, ou le PSVA - Prosthesis Substitution Vision with Audition) permettent essentiellement de collecter des informations sur l'emplacement et la distance des objets, tandis que les systèmes visuo-tactiles (tels que le TVSS), permettent d'accéder à des informations plus complexes de nature optique, telles que forme des objets, profondeur, perspective, et parallaxe de mouvement.

Le principe du TVSS consiste à collecter des informations visuelles à l'aide d'une micro caméra et à les transformer à l'aide d'un système informatique de couplage en images tactiles (patterns de stimulation électro-tactiles de très faible intensité produisant une sensation de pression ou de vibration) transmises au niveau d'une matrice composée de tacteurs placée sur le ventre ou au contact de la langue (cf. Tongue Display Unit - Figure 1) suivant la version utilisée.
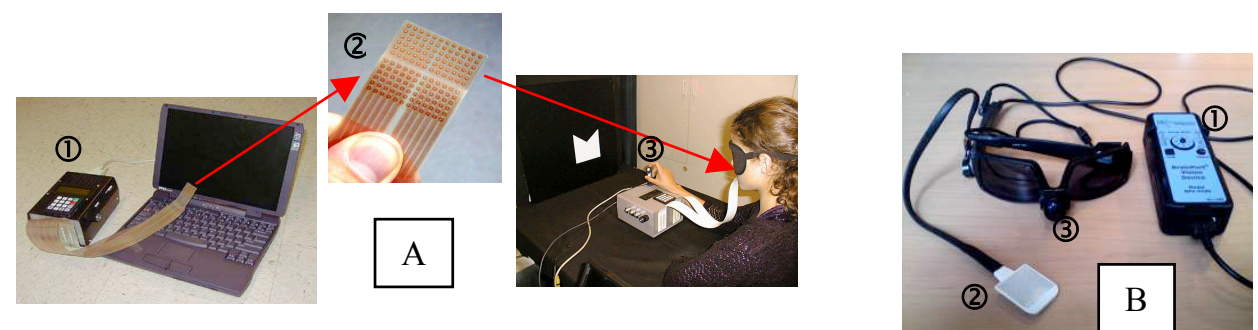

Figure 1: Version TDU du TVSS et ses 3 composants (1)interface informatique/contrôle de l'intensité de stimulation, (2)matrice buccale, (3)micro-caméra). A : version 1998 de Kaczmarek et Tyler; B : version 2010 BrainPort $\AA$ Vision Devive V100. 
Les systèmes visuo-auditifs ont fait l'objet de nombreuses publications dans les années 70 et 80 . Ils suscitent aujourd'hui peu d'intérêt chez les chercheurs et ont rarement été intégrés aux programmes éducatifs de prise en charge pour très jeunes enfants aveugles. En fait, ces systèmes présentent des désavantages majeurs liés à une faible résolution et à la conversion spécifique proposée qui est difficilement transposable à des objets ou formes présentant des propriétés plus complexes que de simples éléments ou points horizontaux ou verticaux. De plus, une autre limitation des systèmes visuo-auditifs est la stimulation du système auditif qui est déjà grandement sollicité chez les personnes aveugles dans leur vie quotidienne et leurs relations avec l'environnement distant, induisant des interférences entre différents types de stimulations auditives. La suppléance sensorielle visuo-tactile présente de nombreux avantages compte tenu du type d'informations auquel le sujet peut accéder, en ne le privant pas de son environnement sonore.

Les dispositifs de suppléance sensorielle de type TVSS, développés depuis les années 1970, ont utilisé la modalité tactile pour transmettre des informations spatiales. Comme c'est le toucher qui suppléante le mieux la vision défaillante [Segond 2008], le toucher étant la modalité la plus redondante avec la vision, et utilisé intensivement par les aveugles pour accéder aux connaissances spatiales et physiques de l'environnement, ce dispositif fait transiter par les voies somesthésiques (cf. sensibilités tactile, thermique et douloureuse du corps) les données apportées habituellement par la vision et c'est donc au bénéfice du toucher que se fait ici la suppléance sensorielle. Ces dispositifs, qui transmettent une image tactile bidimensionnelle projective d'un ou de plusieurs objets tridimensionnels, permettent une perception tactile à distance des objets ; processus qui par conséquent répond également positivement à la problématique de l'autiste précisée ci-dessus. Le développement de telles prothèses sensorielles s'appuie sur la remarquable flexibilité des capacités fonctionnelles des systèmes perceptifs et sur la plasticité cérébrale, définie en termes de capacité adaptative du système nerveux central [Bach-y-Rita $1972 ; 1988 ; 1995]$. Les recherches menées révèlent l'importance des mouvements d'exploration effectués par le sujet (d'avant en arrière, mouvements latéraux, utilisation du zoom, etc..) qui manipule la caméra (permettant de capter les informations visuelles ultérieurement transcrites en images tactiles). Ce dispositif souligne ainsi les liens indissociables qui relient la perception et l'action motrice, liens mis en évidence aujourd'hui dans les principales modalités perceptives, et surtout dans le toucher.

Chez l'adulte, les tout premiers résultats, obtenus avec la première version du TVSS montraient que, sans aucun entraînement, les sujets - aveugles ou voyants aux yeux bandés, manipulant la caméra - étaient capables de percevoir des formes simples, de s'orienter vers elles, et d'indiquer la direction suivie par des cibles mouvantes [Bach-y-Rita 1972]. D'autres études ont montré qu'après 5 à 10 heures d'entraînement, les sujets étaient capables d'identifier des formes géométriques et des objets familiers, d'apprendre des concepts visuels tels que la perspective, la parallaxe de mouvement, l'expansion (looming), ou la focalisation (zooming), et relatifs à des jugements de profondeur [Bach-y-Rita and Hughes 1985; Guarniero 1977; Miletic, Hughes and Bach-y-Rita 1988; Segond, Weiss and Sampaio 2005]. Ils pouvaient découvrir des effets optiques et développer des concepts visuels comme la modification des formes en fonction du point de vue, ou la modification de la taille relative, en fonction de la distance [Segond et al. 2005]. Il a été démontré que l'expérience subjective résultant de ce type de stimulation est comparable à celle de la vision [Bach-y-Rita 1972 ; Sampaio, Maris and Bach-y-Rita 2001 ; Segond et al. 2005]. Ainsi, les témoignages des sujets révèlent que les perceptions sont initialement uniquement associées 
aux stimulations ressenties au niveau de la peau. Mais après entraînement, les sujets perçoivent des objets tridimensionnels localisés dans l'espace extracorporel, face à eux, en oubliant l'origine tactile de l'information [Auvray, Hanneton, Lenay and O'Regan 2005 ; Guarniero 1974 ; Sampaio 1995], reflétant ainsi le phénomène de mise en extériorité des percepts, caractéristique de la suppléance sensorielle visuo-tactile. Ces résultats remettent en question la traditionnelle opposition entre perception tactile et perception visuelle. Comme Proust [1997] le suggère, si les personnes aveugles reçoivent la même information que celle qui induit la vision chez les voyants, on peut considérer alors que les mêmes propriétés invariantes des objets sont extraites par la vision normale et la vision « tactile».

Chez les bébés, l'attrait de la stimulation inhabituelle produite par un dispositif de suppléance sensorielle visuo-tactile (TVSS), considérée comme fournissant des informations optiques aux aveugles par l'intermédiaire de la modalité tactile, a été évalué [23]. Avant de proposer un tel dispositif à des bébés aveugles, il fut nécessaire de démontrer au préalable que ce type de stimulation était plaisant et attractif pour des bébés voyants, en comparaison avec des renforcements visuels et auditifs. Une micro caméra, placée sur le pied gauche ou droit du bébé, convertissait les informations visuelles en données binaires transmises à une matrice placée sur l'abdomen. Les comportements du bébé ont révélé, à travers la recherche active de la stimulation, au cours d'une procédure de conditionnement opérant de l'activité spontanée de battement de pieds, la valeur attractive des stimulations TVSS motivant les interactions du bébé avec son environnement, en sélectionnant les comportements responsables de l'obtention du renforcement. Ces premiers résultats nous permettent de considérer la possibilité de développer des dispositifs pratiques de suppléance visuelle pour de très jeunes enfants [Segond et al. 2005]. Les performances permises par ces dispositifs restent dépendantes d'améliorations technologiques (telles que miniaturisation, résolution vidéo, et connections entre les composants) et de procédures efficaces d'apprentissage en vue d'une utilisation en autonomie et écologique dans l'environnement de la vie quotidienne.

Nos études actuelles menées au niveau comportemental et anatomo-fonctionnel auprès de personnes aveugles congénitales et voyantes contrôles aux yeux bandés se poursuivent avec diverses versions développées d'Interfaces Hommes Machines (IHM) dédiées à la suppléance sensorielle visuo-tactile permettant la stimulation de diverses parties du corps, dont la langue (très sensible et mobile), particulièrement adaptée à la mise au point d'une IHM esthétiquement acceptable et particulièrement efficace. La suppléance sensorielle et la plasticité corticale et cognitive, sont au cœur de nos activités de recherche. Nos études sont menées non pas selon l'unique versant comportemental, à l'origine d'une littérature déjà abondante depuis les années 1970, mais en combinant les approches complémentaires de la psychologie expérimentale et de la neuroimagerie cérébrale fonctionnelle. Le rôle spécifique de l'expérience visuelle, dans ce cadre de recherche, est particulièrement étudié en comparant des sujets aveugles précoces et des sujets voyants aux yeux bandés.

Nos activités de recherche s'inscrivent ainsi dans le contexte théorique de la suppléance sensorielle, et concernent plus particulièrement l'étude de la perception d'informations relatives à l'espace extracorporel sur la base de stimuli «substitutifs", transmis via des systèmes de suppléance sensorielle visuo-tactile, et sur la plasticité cérébrale et comportementale que semble impliquer l'utilisation de ces prothèses sensorielles. Nos études portent sur la perception d'informations de nature optique à travers des procédures d'apprentissage, inspirées par différentes études ayant démontré les possibilités de percevoir 
certaines propriétés de l'espace tridimensionnel via des stimulations vibro- ou électrotactiles. L'un des objectifs majeurs de ces recherches est l'évaluation des effets comportementaux et anatomo-fonctionnels de l'utilisation de ces prothèses sensorielles. Des techniques psychophysiques sont appliquées pour étudier les effets perceptifs et cognitifs de la suppléance sensorielle visuo-tactile et les possibilités de faire l'expérience tactile d'effets optiques (i.e. des patterns de stimulation dont la structure est fortement corrélée avec une stimulation optique [Epstein 1985]) habituellement reliés à la vision. L'évaluation est réalisée en proposant différentes tâches comportementales réalisées au cours d'un apprentissage via un dispositif de suppléance sensorielle. D'autre part, la technique d'imagerie cérébrale par résonance magnétique fonctionnelle (IRMf) est utilisée pour évaluer la plasticité cérébrale, ce qui sous-entend la localisation des régions cérébrales impliquées dans la perception des stimuli substitutifs, et l'évolution des foyers d'activations en lien avec l'apprentissage comportemental réalisé. Ainsi, cette double approche a permis de mettre en évidence deux catégories de résultats dans le domaine de la suppléance sensorielle :

Tout d'abord, au niveau comportemental, de très grandes capacités de discrimination de formes ont pu être obtenues, tout particulièrement avec la version très miniaturisée du TVSS (le TDU ; Tongue Display Unit) qui associe une haute densité des tacteurs et des récepteurs sensoriels tactiles dans l'environnement protégé qu'est la bouche. Ainsi, l'acuité « visuotactile » des personnes aveugles percevant l'information à travers le TDU a été quantifiée, en utilisant un test ophtalmologique standard (la charte de Snellen). Sans aucun entraînement, les adultes voyants aux yeux bandés et les personnes aveugles présentaient une acuité de $1 / 43^{\mathrm{e}}$ (tandis que l'institut Dobelle obtenait, avec un sujet adulte aveugle bien entraîné, une acuité de $1 / 60^{\mathrm{e}}$ à l'aide d'un dispositif très invasif implanté directement dans le cortex occipital [Dobelle 2000]). A la fin de la période d'entraînement, nos sujets ont doublé leur acuité, atteignant $1 / 22^{\mathrm{e}}$ [Sampaio et al. 2001]. De plus, l'entraînement intensif à l'usage du TVSS, avec différents niveaux adaptés et progressifs de difficulté, permettait à chacun des groupes (sujets aveugles congénitaux et voyants contrôle aux yeux bandés) de percevoir des informations spécifiquement visuelles telles que la profondeur, la perspective ou la parallaxe de mouvement. En fait, la suppléance sensorielle constitue la seule voie d'accès pour les personnes aveugles à ce type d'information localisée dans l'espace distant extracorporel. Même à l'aide d'une faible densité de tacteurs (les 64 tacteurs de la version PTD - Pneumatically Tactile Driven system - du TVSS), une procédure d'apprentissage appropriée permet une navigation efficace dans un espace 3D, en radio-contrôlant un robot (Mobile de Couplage Sensori-Moteur) dans un labyrinthe sur la seule base d'informations substitutives visuo-tactiles [Segond et al. 2005]. Un tel résultat présente un impact social et économique potentiel comme le soulignent ces possibilités de faciliter la navigation des personnes aveugles dans le monde réel. De plus, il suggère également qu'il est possible d'implémenter non seulement des aides au déplacement, mais également à la communication y compris à travers des systèmes de type jeux vidéo contribuant à l'implication des personnes aveugles dans tous les domaines de la vie en société, pour une meilleure qualité de vie en lien avec l'accès aux loisirs associés aux développements technologiques.

Au niveau anatomo-fonctionnel et cérébral, des patterns visuels d'activation cérébrale dans des aires habituellement responsables du traitement de la profondeur et du mouvement à partir d'informations visuelles, ont été obtenus après un apprentissage intensif, bien qu'une stimulation tactile était présentée, issue du TVSS (version PTD destinée à une utilisation 
dans l'environnement du scanner IRMf). Ainsi, l'imagerie fonctionnelle suggère que l'information visuelle peut être traitée par la modalité tactile quand une transmission adéquate est effectuée par l'intermédiaire du TVSS. Par ailleurs, il a été démontré, que non seulement pouvait être utilisée en toute sécurité dans l'environnement IRMf la version pneumatique du TVSS (PTD), spécialement constituée d'éléments amagnétiques, mais également la version électronique miniaturisée TDU (matrice de $3 \times 3 \mathrm{~cm}$ avec 144 électrodes explorées par la langue). Ce résultat ouvre très largement de nouvelles perspectives d'investigations scientifiques.

\section{DEFICIENCE VISUELLE ET SUPPLEANCE SENSORIELLE}

La vision est la modalité sensorielle privilégiée pour la découverte et la compréhension de notre environnement, ainsi que pour s'adapter à ses changements. En effet, en l'absence de prise en charge précoce, divers troubles perceptifs et cognitifs apparaissent, pouvant donner lieu à des troubles pathologiques graves au niveau du développement moteur, de l'acquisition du langage, de la représentation spatiale, des interactions parents-enfants, du développement de la personnalité, des interactions avec l'environnement physique et social, de l'autonomie et de la confiance en soi. Ainsi, les troubles de type autistiques apparaissent de manière plus fréquente au sein de cette population; ils concernent 25 à $50 \%$ des enfants aveugles selon les statistiques américaines [Sampaio 1989], contre $0,09 \%$ dans la population générale (cf. circulaire ministérielle du 8 mars $2005^{1}$ ).

Un tel phénomène souligne la nécessité de développer les études sur la suppléance sensorielle visuo-tactile et de concevoir un dispositif embarqué à liaisons non filaires, esthétiquement acceptable et utilisable dans les situations de la vie quotidienne permettant à de jeunes aveugles de détecter la présence et comprendre la nature d'objets distants, et de découvrir les conséquences perceptives de leurs propres actions - une clé pour le développement d'un sentiment d'efficacité et de confiance en soi. La prise en charge précoce des enfants aveugles doit les inciter et leur permettre d'interagir avec leur environnement, en particulier à travers la manipulation d'objets. L'objectif essentiel étant qu'ils puissent contrôler activement leurs interactions par la production d'une corrélation systématique entre un mouvement et ses effets consécutifs. Cette capacité, ainsi que le caractère attractif pour de jeunes bébés des stimulations issues d'un dispositif de suppléance sensorielle visuo-tactile de type TVSS (Tactile Vision Substitution System; prototype à liaisons filaires), ont été récemment scientifiquement démontrés par notre équipe [Segond, Weiss and Sampaio 2007], en lien avec les travaux soulignant les bénéfices du toucher au cours du développement précoce [Segond 2008]. Chez l'adulte aveugle, l'enquête Handicaps-Incapacités-Dépendance ${ }^{2}$, menée récemment en France sur la déficience visuelle, a permis d'analyser les restrictions d'activité occasionnées par le handicap en fonction de sa sévérité. La déficience visuelle réduit l'autonomie; les incapacités sévères concernent de manière dominante la mobilité et les déplacements, ainsi que les tâches ménagères, et les activités de souplesse et de manipulation (se pencher, ramasser un objet sur le sol...). Ces

\footnotetext{
${ }^{1}$ Circulaire du 8 mars 2005. Disponible à l'adresse http://www.cra-rhone-alpes.org/spip.php?article144

2 Enquête HID; Observatoire Régional de la Santé des Pays de Loire, 2005. Disponible à l'adresse www.sante.gouv.fr
} 
incapacités sont déclarées, selon l'enquête HID, par $80 \%$ des aveugles et malvoyants profonds.

Concernant les aides techniques pour la compensation du handicap visuel, celles-ci se révèlent très peu utilisées ( $15 \%$ seulement des déficients visuels en ont à leur disposition), et les besoins dans ce domaine sont très largement insatisfaits. Les seules « aides visuelles » au déplacement dont disposent à l'heure actuelle les personnes aveugles sont la canne blanche (l'ancêtre des dispositifs TVSS) et le chien-guide. Or, celles-ci sont parallèlement très peu utilisées même dans le cas des déficiences les plus sévères. $2 \%$ des déficients visuels déclarent utiliser une canne blanche, et 1/1000 un chien-guide. Des dispositifs technologiques de détection d'obstacle à distance de type télémètre laser ou proximètre infrarouge (Tom Pouce, Télétact ${ }^{3}$ ) peuvent être associés à la canne blanche pour améliorer les performances de navigation des personnes aveugles. Parallèlement, les aides à la communication (utilisant le braille, la synthèse vocale, les lecteurs d'écran) destinées également aux personnes les plus déficientes, se sont développées ces 10 dernières années afin de permettre, avec le développement des nouvelles technologies de l'information et de la communication (NTIC), et notamment de l'internet, une amélioration de l'accessibilité à l'information (techniques nécessitant une phase d'apprentissage). Les techniques reposant sur le braille sont peu exploitables compte tenu du fait qu'il existe une grande partie des personnes aveugles qui rencontrent des difficultés dans la lecture et la compréhension du braille $^{4}$. Aussi, utiliser conjointement un ordinateur (via son interface) et un assistant technique (lecteur d'écran et plage braille) représente une double tâche impossible à gérer pour la plupart des personnes. Ainsi, ceux qui veulent interagir avec un ordinateur se servent essentiellement du synthétiseur vocal. Actuellement, si ces techniques et interfaces d'ordinateurs sont majoritairement utilisées par les aveugles profonds (les technologies informatiques se révélant particulièrement intéressantes en cas de déficience visuelle majeure), elles sont peu répandues (moins de $2 \%$ des personnes aveugles -1000 personnes déclarent y avoir accès). Or il est important de souligner que si le recours à ce type d'aide est peu fréquent, les besoins réels apparaissent quant à eux beaucoup plus importants : 24.000 déficients visuels n'utilisent pas d'interfaces d'ordinateurs mais déclarent en avoir besoin. Et les besoins sont très vraisemblablement plus importants en raison du fait que les déficients visuels ont peu connaissance de l'existence même des aides visuelles. Le manque d'accessibilité numérique des environnements technologiques contribue à cette méconnaissance; les personnes aveugles (même munies de lecteurs d'écran reliés à des plages braille ou de synthétiseurs vocaux) ne pouvant consulter et parcourir les sites web. Cette difficulté est à l'origine de la création de la WAI (Web Accessibility Initiative) lancée en 1997. Des initiatives intéressantes ont vu le jour pour le développement d'interfaces comme les projets Tactos $^{5}$ et TIM (Tactile Interactive Multimedia) ${ }^{6}$. Cette dynamique se

\footnotetext{
${ }^{3}$ Tom Pouce et Télétact. Disponibles à l'adresse http://www.lac.u-psud.fr/teletact/index-teletact.htm

4 « Dans les faits, beaucoup des personnes atteintes de cécité ont des notions de braille. Elles parviennent, ainsi, à déchiffrer quelques caractères, prendre des notes et étiqueter certains objets afin de les reconnaître. Très peu sont cependant à même de lire un document en entier rapidement. Cette maîtrise du braille est pratiquement réservée aux aveugles de naissance ou de jeunesse. On estime que $10 \%$ à $15 \%$ seulement des non voyants la possèdent, soit environ 8.000 personnes, qui représentent à peine $2 \%$ des 500.000 déficients visuels graves, et ce pourcentage est en constante diminution. »http://www.air-asso.org/deficience_visuelle_autonomie_braille.htm

5 Tactos: dispositif du CRED. Disponible à l'adresse http://www.utc.fr/cred/index.php?page=ressources\&hl=fr_FR

${ }^{6}$ TIM. Disponible à l'adresse http://inova.snv.jussieu.fr/tim/tim.php?ref=adapt01
} 
voit soutenue par la publication récente d'un décret d'application ${ }^{7}$ sur l'accessibilité numérique des sites E-administration (dans le prolongement de la loi égalité des chances de février 2005) qui fixe un délai maximal de deux ans pour rendre accessible les sites en ligne des établissements publics dépendant de l'Etat. Les besoins ne sont pas mieux satisfaits en institution qu'à domicile, traduisant par là-même la méconnaissance des aides visuelles non seulement par les utilisateurs potentiels, mais également par les professionnels de santé amenés à intervenir dans le champ du handicap visuel. Cette méconnaissance concerne autant l'évaluation des besoins des déficients visuels, que le bénéfice potentiel de ces aides, et qui plus est les procédures d'apprentissage requises par ce type de dispositif.

A ce manque de connaissance et de formation dans le domaine des aides visuelles s'ajoute également, parmi les freins à l'utilisation de ces dispositifs, le prix des aides visuelles, d'autant plus élevé qu'elles ont recours à une technologie électronique ou informatique. L'exemple Québécois illustre bien ce phénomène dans la mesure où la gratuité des aides visuelles favorise considérablement leur diffusion (dans le cadre d'un programme d'aides visuelles mis en place pour les malvoyants depuis 1977, avec prêt de matériel). Or ces coûts dépendent également de l'importance du marché ciblé. La conception recherchée dans le cadre de nos activités de recherche d'une base technologique commune à la mise au point d'un dispositif de type TVSS, avec des interfaces modulables permettant une utilisation auprès d'un plus large panel de population (bébés, enfants, adultes aveugles et/ou autistes) constitue des éléments de réponse à ce problème. D'autres éléments déterminants pour l'accès à ces dispositifs sont d'ordre politique et concernent en particulier la prise en charge des matériels informatiques pour déficients visuels dans le cadre des prestations légales. Or, un dispositif pour la vie autonome a récemment été mis en place et s'est progressivement généralisé depuis 2000 à l'ensemble des départements français venant compléter les dispositifs de financements complémentaires pouvant être obtenus auprès de différents organismes (Association de Gestion du Fonds pour l'Insertion Professionnelle des Personnes Handicapées, Fonds pour l'Insertion des Personnes Handicapées, Maison Départementale des Personnes Handicapées, conseils généraux, plan Handiscol, assurances et mutuelles...). Ces nouvelles dispositions constituent une avancée dans le domaine de l'accessibilité aux aides visuelles.

Enfin, il faut également souligner parmi les facteurs limitant actuellement l'accès aux aides visuelles la multiplicité des circuits de distribution, qui diffèrent en fonction de la nature des aides. La diffusion des aides au déplacement et à la communication est tout particulièrement insatisfaisante, contribuant à la méconnaissance de l'existence des potentialités offertes par les technologies nouvelles et innovantes.

\section{NOUVELLE PERSPECTIVE : TVSS ET AUTISME}

L'autisme est un trouble envahissant du développement (TED) qui se caractérise par des dysfonctionnements dans les interactions sociales, des difficultés de communication, de symbolisation et des comportements bizarres et stéréotypés. On connaît actuellement l'existence de facteurs génétiques et d'altérations précoces du système nerveux central pouvant être associés à ces troubles [Tourette 2006]. La contribution de facteurs environnementaux reste sujette à discussion.

\footnotetext{
${ }^{7}$ Décret d'application n²009-546 du 14 mai 2009
} 
L'observation quotidienne des personnes avec autisme sévèrement déficitaires met clairement en évidence un mode de fonctionnement " sensoriel », basé sur un usage aberrant des fonctions sensorielles. Ces observations sur le terrain rejoignent les concepts de seconde peau [Bick 1986], de holding [Winnicott 1971 - 2006], de démantèlement du système perceptuel [Meltzer, Brenner and Hoxter 1975] ou de trous noirs [Tustin 1986]. De façon globale, les perceptions proximales (le toucher) sont préférées aux perceptions distales (la vision et l'audition). Les sensations vibratoires sont souvent recherchées. Les comportements de balancement du corps, de reniflage sont fréquemment observés. Autant de bizarreries comportementales qui signent la clinique autistique. Les perspectives analytiques y voient des troubles dans la constitution psychique de l'être, allant de la naissance psychologique à la constitution du sujet pensant et parlant. Les différentes théories détaillent ensuite l'altération des processus d'identification primaire à travers les différents concepts mis en avant. Ainsi Tustin [1986] considère l'hypothèse d'une non constitution de l'enveloppe psychocorporelle de l'enfant comme l'élément majeur pouvant expliquer les signes autistiques. Winnicott [1971 - 2006] souligne à ce sujet le rôle de l'enveloppement de l'enfant prodigué par les parents lors du portage (holding) et des manipulations de l'enfant lors de soins quotidiens (handling), exerçant une véritable fonction de contenance des angoisses psychiques de l'enfant ou système de parexcitation, qui ferait défaut dans le cas de l'autisme. Cette absence de première ébauche psychocorporelle fera que pour l'enfant autiste, l'autre est perçu sans accrochage, les sensations sont non mémorisées et stabilisées (Moi-sensation) dans le corps. Ce qui crée des angoisses primitives, des trous noirs, c'est-àdire des lieux corporels où les sensations sont éprouvées régulièrement et qui font trou dès que cesse l'excitation sensorielle. Les exemples de trous noirs sont nombreux : le dos, d'où les stéréotypies de balancement qui sont des stimulations dorsales; la bouche «molle » des autistes, marquant une hypotonie musculaire et un déficit sensoriel. Ainsi l'absence de sphinctérisation des lèvres sur le couvert (fourchette raclée, absence de préhension des lèvres, aliments déposés sur la langue) ou la rareté de la mastication sont régulièrement observées. Les idées avancées, entre autres par ces auteurs, permettent de comprendre certains troubles du comportement comme autant de tentatives pour retrouver les sensations, pour lutter contre les trous noirs; les stimulations corporelles quasi-permanentes sont perçues comme des tentatives de créer une véritable seconde peau. Cette idée peut se traduire à travers la métaphore d'une véritable tentative de «tactiliser» les sensations pour rendre compte de tous ces comportements autour de la sensorialité.

Les sens sont tactilisés par les personnes autistes à partir 1. de la vision, en privilégiant la vision périphérique car la vision fovéale est celle qui permet d'accéder à la spatialisation et la tridimensionnalité (les distances sont évaluées et les objets sont perçus comme des nonMoi) ; 2. de la chémoréception oro-pharyngée, en utilisant le système olfactif trigéminale ; l'un des 3 systèmes olfactifs chez l'homme, responsable des sensations fortes, piquantes, irritantes. De telles sensations illustrent la «tactilisation» olfactive voire gustative; 3. de l'audition en recherchant les composantes vibratoires de basses fréquences traduisant une tactilisation des stimulations sonores (la composante auditive du son devenant alors une composante vibratoire tactilement perçue). Ces considérations, issues des interprétations de type psychanalytique, n'induisent rien quant à l'étiologie de l'autisme, mais peuvent contribuer à la compréhension de ce qui se joue chez la personne autiste. Le meilleur argument en faveur de ce type de lecture est la relative efficacité des actions menées sur le terrain. Toutes les personnes qui ont en charge les personnes autistes connaissent 
l'importance des techniques d'enveloppement (indépendamment du packing ${ }^{8}$ visant à lutter contre les automutilations par l'enveloppement du corps de l'autiste) et le rôle apaisant de tout ce qui concerne la question de la contenance pour la personne autiste. Cette efficacité est relative dans le sens où elle n'est jamais que temporaire.

L'entrave à la constitution du psychisme de l'enfant, qui est l'hypothèse centrale, n'est en rien l'origine des troubles; elle n'en est que la conséquence. L'origine relève probablement de dysfonctionnements du système nerveux. Des lésions cérébrales, même si elles ne sont pas toujours identifiées, créeraient des difficultés inhérentes à la constitution du psychisme. Dans cette perspective, l'approche neuropsychologique issue des neurosciences, les approches psychanalytiques et comportementalistes sont pour nous complémentaires. En effet, certaines de ces idées peuvent s'articuler avec des données issues de la neuropsychologie et des neurosciences. Les travaux en imagerie cérébrale commencent à se multiplier malgré les difficultés méthodologiques inhérentes à ce public. Les résultats obtenus en imagerie cérébrale (voir par exemple [Boddaert and Zilbovicius 2002]) confirment partiellement l'idée centrale d'un fonctionnement sensoriel aberrant mis en avant par l'observation clinique et repris par des psychanalystes. Ceci montre bien la complémentarité des points de vue et la nécessaire concertation des approches et des idées.

L'intérêt à utiliser des dispositifs de type TVSS chez les personnes autistes (avec retard mental grave associé) n'a jamais été envisagé jusqu'à présent. Or, cet intérêt est souligné par le fait qu'elles affectionnent tout particulièrement les modalités tactile et vibratoire sous réserve qu'elles en soient à l'origine : ce sont des sensations qu'elles recherchent et qu'elles se créent. Si elles n'aiment pas être touchées, ce sont elles qui initient le contact tactile. L'idée de la tactilisation des sens permet d'illustrer cette attirance qu'ont les personnes autistes pour ce type de stimulations. Ce sont précisément ces stimulations que permettent les dispositifs TVSS. Les systèmes TVSS représentent un outil thérapeutique particulièrement séduisant dans la mesure où ils fournissent non seulement des informations sur la localisation des objets dans l'espace, mais également sur la structure des objets [Bachy-Rita, Kaczmarek and Tyler 2003]. Ces mêmes bénéfices pourraient être mis à profit auprès de personnes autistes. L'utilisation de ces appareils occasionne des sensations tactiles sur la peau ou sur la partie du corps où est posée la matrice. De plus l'usage de la caméra permet une utilisation en boucle fermée : ce sont bien les mouvements de la caméra manipulée par la personne qui créent les sensations correspondantes. Cela offre la possibilité au sujet de maîtriser totalement les stimulations. Cette maîtrise est fondamentale pour les sujets autistes, car ils agiront au lieu de subir. Ils seront acteurs de leur environnement; ils pourront même en être investigateurs et peut être produire des comportements exploratoires. Seule la réalisation concrète sur le terrain pourra dire quelles peuvent en être toutes les conséquences et toutes les perspectives.

Il existe une autre raison importante pour utiliser ces dispositifs avec la population autistique. De toute évidence la perception visuelle de l'environnement physique, donc spatial, pose difficulté. Tout nouvel environnement génère angoisse et stéréotypies. Classiquement, la préférence en faveur de la vision périphérique est interprétée comme un

\footnotetext{
${ }^{8}$ Enveloppement serré, humide (le plus souvent froid), suivi d'un réchauffement, réalisé avec des draps et des couvertures au cours de séances répétées durant chacune 45 minutes, réservé à des cas gravissimes, le plus souvent en dernier recours, notamment en cas d'auto ou hétéroagressivité et d'agitation extrême (cf . avis du Haut Conseil de la Santé Publique publié en 2010).
} 
renoncement à la spatialité, à la vision tridimensionnelle. Voir en perspective, en volume, est anxiogène et cette idée est traduite par la métaphore suivante : l'autiste est fâché avec les distances et les volumes; il préfère les sensations corporelles, aussi pour leur aspect de bi dimensionnalité. Les dispositifs TVSS semblent alors offrir une situation idéale pour un autiste : accéder à une perception distale au travers d'une sensation tactile ou encore accéder à une perception de l'espace et des volumes à travers une perception bidimensionnelle. Du point de vue développé ici, cette situation semble réaliser un rêve impossible pour la personne souffrant du syndrome autistique.

\section{CONCLUSION}

Sur le plan technique, l'enjeu important de nos activités consiste à coupler la micro caméra embarquée du dispositif TVSS (intégrée soit à une tétine chez le bébé, soit à une branche de lunettes pour les enfants/adultes, ou encore à un bandeau frontal) avec un dispositif sans fil d'électrostimulation (linguale ou abdominale), lui-même interfacé par liaisons non filaires avec le système microinformatique de couplage embarqué (de type mini-PC ou NotePad) qui traite par voie logicielle les informations optiques transmises par la micro caméra pour les renvoyer sous forme d'image tactile au niveau de la matrice de tacteurs. La seconde étape de l'interfaçage, au profit de l'aide à l'autonomie des personnes aveugles, consistera à coupler le TVSS avec un logiciel PC présentant l'environnement urbain numérisé en 3D de la personne aveugle, permettant non seulement la découverte de dimensions habituellement inaccessibles de l'espace (éléments architecturaux - cf. hauteur des murs, toitures...configuration globale de l'espace urbain), d'informations optiques (profondeur, perspective...), et la familiarisation à un trajet permettant ensuite à la personne aveugle de se rendre où bon lui semble, équipée du dispositif embarqué de Réalité Augmentée: l'ECOVISIOTACT.

Quant à la prise en charge des personnes autistes, le bénéfice de celle-ci repose sur la création d'un environnement sécurisant à la fois par l'adéquation de l'environnement psychophysique à leurs spécificités sensorielles et par l'accordage affectif de l'intervenant dans un souci de non directivité et de libre choix. L'impact d'une telle approche doit faire l'objet d'une évaluation en définissant préalablement le profil sensoriel individuel caractérisant le fonctionnement sensori-moteur et émotionnel atypique de la personne autiste, en fonction des modalités sensorielles impliquées et des possibles intégrations ou dissociations entre ces dernières. Cette approche nous conduira à évaluer la pertinence de l'utilisation innovante d'un dispositif de suppléance sensorielle visuo-tactile en tant que stratégie innovante d'intégration sensorielle et de régulation émotionnelle pour les individus en questions [Bellusso, Desnos, Maris, Salman and Segond 2009; Desnos, Maris and Segond 2009 ; Desnos and Segond 2009].

\section{REMERCIEMENTS}

Nous remercions tout particulièrement le Conseil Scientifique de l'Université de Strasbourg, le Conseil Régional d'Alsace, M. Albecker et la Fondation Sonnenhof (Bischwiller), M. Dietrich et le Foyer d'Accueil Médicalisé Pierre Valdo (Marmoutier), ainsi que Mme Birki et l'IME Les Rosiers Blancs (Saverne).

\section{RÉFÉRENCES}


Auvray, C., Hanneton, S., Lenay, C. and O'Regan, J.K. 2005. There is something out there: distal attribution in sensory substitution, twenty years later. Journal of Integrative Neuroscience, Vol. 4, No. 4, pp. 505-521.

Bellusso, P., Desnos, Y, Maris, S., Salman, S. and Segond, H. 2009. Dispositif Ecologique Embarqué de Suppléance Sensorielle Visuo-Tactile pour personnes aveugles et/ou autistes sévèrement déficitaires. Sciences et Technologies Cognitives, Colloque de Prospectives, Paris, 23 oct.

Bick, E. 1986. Further considerations on the function of the skin in early object relations. British journal of psychotherapy, 2, pp. 292-299.

Bach-y-Rita, P. 1972. Brain Mechanisms in Sensory Substitution. Academic Press, New York.

Bach-y-Rita, P. 1988. Brain Plasticity. In J. Goodgold (ed.), Rehabilitation Medecine. Mosby, pp. 113-118.

Bach-y-Rita, P. 1995. Nonsynaptic diffusion, neuro-transmission and late brain organization. Demos Publications, New York.

Bach-y-Rita, P. and Hughes, B. 1985. Tactile vision substitution: some instrumentation and perceptual considerations. In D. Warren \& E. Strelow (eds.) Electronic spatial sensing for the Blind. Martinus-Nijhoff, Dordrecht, pp. 171-186.

Bach-y-Rita, P., Kaczmarek, K.A. and Tyler, B. 2003. A tongue-based tactile display for portrayal of environmental characteristics. In L. Hettlinger \& M. Haas (eds.), Psychological issues in the design and use of virtual and adaptive environment, Lawrence Erlbaum, Mahwah, N.J., pp. 169-186.

Boddaert, N. and Zilbovicius, M. 2002/1. L'imagerie cérébrale et l'autisme infantile. Enfance, Vol. 54, pp. 10-20.

Brown, J. S. and Duguid, P. 2002. The Social Life of Information. Harvard Business School Press. 356 pages.

Circulaire du 8 mars 2005. Disponible à l'adresse : http://www.education.gouv.fr/bo/2005/15/SANA0530104C.htm

Desnos, Y. and Segond, H. 2009. Substitution sensorielle visuo-tactile et prise en charge thérapeutique des personnes avec autism et retard mental severe associé. CJCSC'09, Colloque des Jeunes Chercheurs en Sciences Cognitives, Toulouse, 9-11 juin.

Desnos, Y., Segond, H. and Maris, S. 2009. Sensory integration devices as a new modality of therapeutic care for autistic people with mental retardation. Innovative Research In Autism, Tours, April 15-17.

Dobelle, W.H. 2000. Artificial Vision for the Blind by Connecting a Television Camera to the Visual Cortex. ASAIO Journal.

Epstein, C.M. 1985. Transmodal perception and amodal information. In D. Warren \& E. Strelow (eds.) Electronic spatial sensing for the Blind. Martinus-Nijhoff, Dordrecht, pp. 421-430.

Enquête HID ; Observatoire Régional de la Santé des Pays de Loire, 2005. Disponible à l'adresse www.sante.gouv.fr

Guarniero, G. 1974. Experience of tactile vision. Perception, Vol. 3, pp. 101-104.

Guarniero, G. 1977. Tactile Vision: A personal view. Journal of Visual Impairment and Blindness, No. 71, pp. 125-130. 
Lenay, C., Gapenne, O., Hanneton, S., Marque, C. and Genouel, C. 2000. La substitution sensorielle: limites et perspectives. In Y. Hatwell, A. Streri, \& E. Gentaz (eds.), Toucher pour connaître. Psychologie cognitive de la perception tactile manuelle. Presses Universitaires de France, Paris, France, pp. 287-306. Traduction anglaise : Touch for Knowing. John Benjamins Publishers, Amsterdam, 2003.

Meltzer, D., Brenner, J., Hoxter, S., 1975. Exploration in Autism. Roland Harris Trust, Clunie Press. trad. fr. Explorations dans le monde de l'autisme. Payot, Paris, 1980.

Miletic, G., Hughes, B. and Bach-y-Rita, P. 1988. Vibrotactile Stimulation: An Educational Program for Spatial Concept Development. Journal of Visual Impairment and Blindness, No. 82, pp. 366-370.

Proust, J. 1997. Perception et Intermodalité : Approches Actuelles de la Question de Molyneux. Presses Universitaires de France, Paris.

Sampaio, E. 1989. L'autisme infantile: le cas de l'enfant aveugle. Réflexions méthodologiques. Psychologie Médicale, No. 21, pp. 2020-2024.

Sampaio, E. 1995. Les substitutions sensorielles adaptées aux déficits visuels importants. In A.B. Safran \& A. Assimacopoulos (eds.), Le déficit visuel. Des fondements neuropsychologiques à la pratique de la réadaptation. Masson, Paris, France, pp. 197211.

Sampaio, E. and Dufier, E. 1988. Suppléance sensorielle électronique pour les jeunes enfants aveugles. Journal Français d'Ophtalmologie, No. 11, pp. 161-167.

Sampaio, E., Maris, S. and Bach-y-Rita, P. 2001. Brain plasticity: "visual acuity" of blind persons via the tongue. Brain Research. No. 908, pp. 276-280.

Segond, H. 2008. Le Toucher en Développement: Perception Tactile et Continuité Transnatale. In G. Chasseigne (eds.), Cognition, Santé et Vie Quotidienne, Vol. 1, Publibook Université, Coll. Sciences Humaines et Sociale/Psychologie Cognitive, Reims, pp. 75-108.

Segond, H., Weiss, D. and Sampaio, E. 2005. Human navigation via a visuo-tactile sensory substitution system. Perception, Vol. 34, pp. 155-164.

Segond, H., Weiss, D. and Sampaio, E. 2007. A proposed Tactile-Stimulation System for Infants Who Are Blind Tested on Sighted Infants. Journal of Visual Impairment and Blindness, Vol. 1, No. 103, pp. 32-43.

Tactos: dispositif du CRED. Disponible à l'adresse

http://www.utc.fr/cred/index.php?page=ressources\&hl=fr_FR

TIM. Disponible à l'adresse

http://inova.snv.jussieu.fr/tim/tim.php?ref=adapt01

Tom Pouce et Télétact. Disponibles à l'adresse

http://www.lac.u-psud.fr/teletact/index-teletact.htm

Tourette, C. 2006. Evaluer les enfants avec déficiences ou troubles du développement. Dunod, Paris.

Tustin, F. 1986. Les états autistiques chez l'enfant. Seuil, Paris.

Winicott, D. 1971, 2006. Jeu et Réalité, l'espace potentiel. Gallimard folio, Paris.

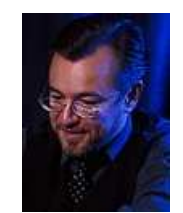

Hervé Segond est Maître de Conférences HDR en Psychologie du Développement à l'Université de Strasbourg où il enseigne le développement normal et psychopathologique de l'enfant au niveau psychomoteur et cognitif depuis la vie fœtale à l'âge scolaire. Il est également Directeur de la Formation 
en Master Professionnel de Psychologie Clinique Développementale « Evolution, Involution et Handicap ». Membre du Comité Directeur du laboratoire de psychologie clinique et psychopathologie (SCLS EA 3071), et responsable de l'équipe de Psychologie du Développement, ses travaux de recherche portent principalement sur le toucher en développement au niveau comportemental et anatomo-fonctionnel, suivant deux axes : a. les origines et le développement de la spécialisation perceptivo-motrice manuelle, ainsi que les troubles de la latéralité en lien avec les troubles des apprentissages et du développement; $\mathbf{b}$. la perception tactile d'informations optiques à l'aide de dispositifs technologiques innovants de suppléance sensorielle visuo-tactile à destination des personnes aveugles, et d'intégration sensorielle au profit de la prise en charge des personnes autistes.

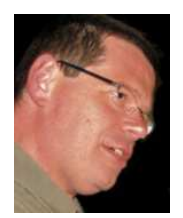

Stéphane Maris est neuropsychologue au Foyer d'Accueil Médicalisé Pierre Valdo (Marmoutier), unité de la Fondation Sonnenhof de Bischwiller (BasRhin), ainsi qu'à l'Hôpital de jour Gériatrique de Wissembourg. Il effectue des prises en charge thérapeutiques auprès d'adultes autistes sévèrement déficitaires et d'adultes polyhandicapés, ainsi que des bilans neuropsychologiques auprès de personnes âgées avec prise en charge de groupes de stimulation cognitive. Docteur en Psychologie, il a mené ses recherches dans le domaine de la suppléance sensorielle visuo-tactile à l'Université de Strasbourg auprès de personnes aveugles. Il est également chargé d'enseignements à l'Université de Strasbourg ainsi qu'auprès des IFSI et de la FEETAC, sur les thèmes de l'autisme (approche cognitiviste et liens avec le cheval) et de la sensorialité des personnes polyhandicapées.

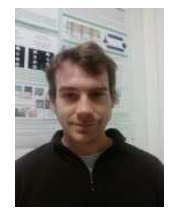

Yves Desnos est Psychologue et Doctorant de l'Université de Strasbourg rattaché au laboratoire de Psychologie Clinique et Psychopathologie SCLS/EA3071. Ses activités de Recherche, financées par le Conseil Régional d'Alsace, sont menées dans l'équipe de Psychologie du Développement sous la direction d'Hervé Segond, en partenariat avec le F.A.M. Pierre Valdo (Marmoutier) sur le thème : prise en charge des personnes autistes sévèrement déficitaires et suppléance sensorielle visuo-tactile; une lecture de l'autisme alliant Psychologie Cognitive du Développement, Neurosciences et Psychanalyse.

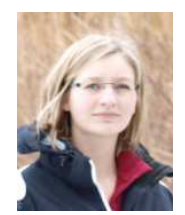

Perrine Bellusso est Psychologue et Doctorante Contractuelle de l'Université de Strasbourg avec Mission d'Enseignement, rattachée au laboratoire de Psychologie Clinique et Psychopathologie SCLS/EA3071. Ses activités de recherche sont menées dans l'équipe de Psychologie du Développement sous la direction d'Hervé Segond, en partenariat avec l'AAPEI et l'IME Les Rosiers Blancs de Saverne, sur le thème : perception et intégration sensorielle chez l'enfant autiste; approche cognitivo-comportementale des bénéfices d'une thérapie sensorielle. 